\title{
A STUDY ON PERSONA BASED MARKETING USING FACE DETECTION
}

\author{
Siddaiah M \\ Department of computer science and engineering \\ Presidency university, Bangalore, India. \\ Ranjitha J \\ Department of computer science and engineering \\ Presidency university, Bangalore, India.
}

\begin{abstract}
The system is produced for conveying a simple and a safe method of doing computerized advertising. The product first catches a picture of all the approved people at the hour of enlistment and stores the data into database. The system at that point stores the picture by mapping it into a face organize structure. In view of visitor face, distinguish the visitor and classify the visitor dependent on Persona.
\end{abstract}

\section{Keywords - Face recognition, Database}

\section{INTRODUCTION}

In present day life, we see more procedures of biometric highlights recognition have been utilized to our encompassing life, particularly the applications in telephones and laptops. These biometric recognition methods contain face recognition, unique mark recognition and iris recognition. Our work centers around the face recognition issue and uses a profound learning technique, to fathom it. Dataset is utilized for preparing and testing which gets a significant outcome. What's more, we additionally test our framework on other face dataset, which likewise has a high precision on the recognition.

\section{METHODOLOGY}

\section{A. FACE RECOGNITION}

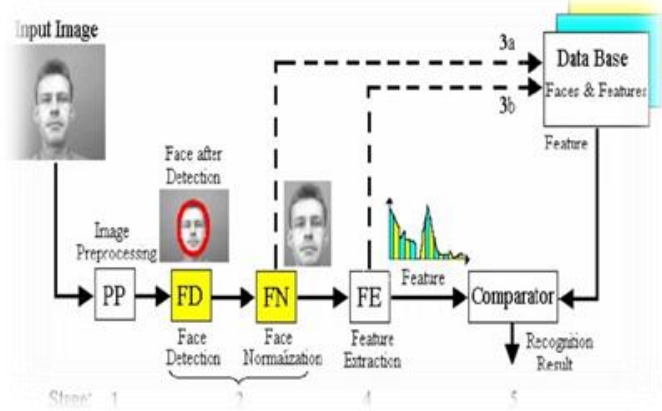

Figure No 1. Face Recognition process
Presilla Jenifer A

Department of computer science and engineering Presidency university, Bangalore, India.

Rakesh M

Department of computer science and engineering Presidency university, Bangalore, India.

\section{IMAGE PREPROCESSING:}

When taking care of a face recognition issue or other face issue, we frequently do the face image pre-processing first, in light of the fact that the original training data isn't reasonable for the training method. For the most part, we decide to do face detection, face tracking, face cropping and face alignment. Along these lines, when goes to the face recognition issue, it is important to take care of those issues before preparing.

\section{FACE DETECTION AND FACE NORMALIZATION:}

Face identification is a computer technology that decides the areas and sizes of human faces in digital images. It distinguishes face and overlooks whatever else, for example, structures, trees and bodies. Face detection can be viewed as an increasingly broad instance of face localization. Scientists consistently accept that face detection is the main undertaking to find the human face among a great deal of different articles in a solitary picture. Furthermore, when countenances could be found precisely in any scene, the recognition step a while later would not be so muddled. After the face detection stage, it regularly goes to the face normalization progress to normalize faces from the first preparing face images and afterward do the face alignment. The goal of face alignment is to restrict the component focuses on face images, for example, the shape purposes of eye, mouth and blueprint. Face alignment $\mathrm{t}$ is basic to many face processing applications including face recognition, modelling and synthesis. What's more, subsequent to resizing, we get the information of our model.

\section{FEATURE EXTRACTION:}

Feature extraction as a rule happens following face detection and can be considered as one of the most significant stages in face recognition systems, as their adequacy is needy upon the nature of the extricated features. This is on the grounds that facial landmarks and fiducial points distinguished by a given system decide how precisely includes are spoken to. 
Conventional fiducial point locators are model-based, while numerous ongoing methods are cascaded regression base.

\section{B. DATABASE}

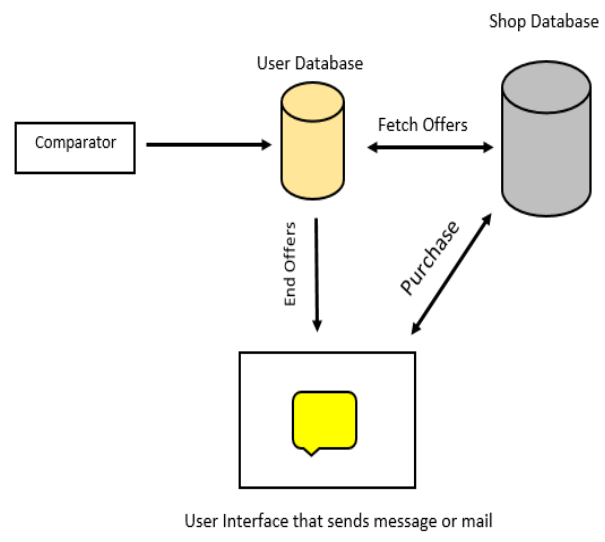

Figure No 2. Database process

In the database we will store the examples of images of individual. On the off chance that the information which we have gathered and which we have been put away in the database ought to be same. the comparator will check whether the examples are coordinating with our database and in the event that it matches, at that point we will get the message face is recognized else we would get the message as " unknown ".

In the event that the face is recognized, at that point it will be put away in the client database from that we gather all the information and there will be of past records

And, from the shop database we will gather the insights about offers or the occasions which are going on in the specific shops at present and afterward send it to the client with the goal that they will come to think about the offers or occasions and even about when the offers will end in shop. And, as indicated by the client past record we will send the clients the message as needs be.

Subsequent to sending the message if the clients are keen on a specific thing, they will be redirected to a shop sites and they can arrange the thing. And, these procedures occur between the clients and the retailer.

\section{FUTURE SCOPE}

Developing web application, where the shop people can insert their offer details based on categories, then the inserted data will be distributed to the visitors database based on the matched visitors who has mentioned their interests as these categories, Then whenever the visitor visits the mall his face will be recognized and then he will be getting mail detailed about that offer

\section{CONCLUSION}

The recommendations based on visitors likes would be more convenient to people in their busy schedule. With the help of face recognition, we implemented a recommendation system that include process like image pre-processing, detection, normalization and feature extraction.

Whenever the registered visitor enters into the mall camera recognize his/her face collect the information related to that particular person. Suggestion will be sent via email based on information collected (previous purchases). If they order through the message sent to them, provide options of delivering items at the entrance of the mall. When visitor goes to collect ordered item, shop authenticate the person through facial recognition and allow to collect ordered items.

\section{REFERENCE}

[1] H. Kim, S. H. Lee and Y. M. Ro,(2015) "Face Image Assessment Learned with Objective and Relative Face Image Qualities for Improved Face Recognition," 2015 IEEE international conference on image processing, QC, 2015, (pp. 4027-4031), doi: 10.1109/ICIP.2015.7351562.

[2] J. Dhamija, T. Choudhury, P. Kumar and Y. S. Rathore, (2017) "An Advancement towards efficient face recognition using live video feed: "For the Future" Odisha, 2017,( pp. 53-56).doi: 10.1109/CINE.2017.21.

[3] S. R. Dubey and S. Mukherjee,(2018) "A Multi-Face Challenging Dataset for Robust Face Recognition," 2018 15th International Conference on Control, Automation, Robotics and Vision (ICARCV), Singapore, 2018,(pp. 168-173), doi: 10.1109/ICARCV.2018.8581283.

[4] Pravin Gopalrao Sarpate, Ramesh R. Manza .(2019),” Face Recognition Using HOG and Different Classification Techniques".

[5] Abad Jr, Bienvenido. (2017). Proposed Image Preprocessing Techniques for Face Recognition Using OpenCV.

[6] Dhara Rathore1, Gaurav Kumar Das2, Dr. Akash Saxena, (2020)," Concept Of Face Recognition: A Review" International Journal For Technological Research In Engineering Volume 7, Issue 5, January-2020.

[7] Sharmila, R. Sharma, D. Kumar, V. Puranik and K. Gautham, (2019) "Performance Analysis of Human Face Recognition Techniques," (pp.1-4), doi: 10.1109/IoTSIU.2019.8777610.

[8] M. S. I. Sameem, T. Qasim and K. Bakhat, (2016) "Real time recognition of human faces," 2016 Conference on Open Source Systems \& Technologies (ICOSST), Lahore, 2016, (pp. 62-65), doi: 10.1109/ICOSST.2016.7838578. 
[9] R. Samet and M. Tanriverdi, (2017) "Face RecognitionBased Mobile Automatic Classroom Attendance ManagementSystem,"(pp.253to256),doi:10.1109/CW.201 7.34.

[10] Ya Wang, Tianlong Bao, Chunhui Ding and Ming Zhu, (2017) "Face Recognition in realworld surveillance videos with DeepLearning method," 2017,(pp.239 243), doi:10.1109/ICIVC.2017.7984553.

[11] Guo, Guodong \& Zhang, Na. (2018). What Is the Challenge for Deep Learning in Unconstrained Face Recognition? (pg.436-442). 10.1109/FG.2018.00070.

[12] Salhi, A.I., Kardouchi, M., Belacel, N.: Fast and efficient face recognition system using random forest and histograms of oriented gradients. In: BIOSIG 2012, (pp. 1-11) (2012). 